\author{
S. Magrini, D. Barreca, L. Neri, A. Iacomino, F. Barbarese, S. Superchi \& \\ L. Zucconi
}

\title{
Seed germination protocols for six dune species from the Tyrrhenian coast (central Italy)
}

\begin{abstract}
Magrini, S., Barreca, D., Neri, L., Iacomino, A., Barbarese, F., Superchi, S. \& Zucconi, L.: Seed germination protocols for six dune species from the Tyrrhenian coast (central Italy) [In Magrini, S. \& Salmeri, C. (eds), Mediterranean plant germination reports - 1]. Fl. Medit. 29: 293-302. 2019. https://doi.org/10.7320/FlMedit29.293

Successful germination protocols for the following six dune species are presented: Centaurea sphaerocephala subsp. sphaerocephala, Jacobaea maritima subsp. maritima, Matthiola sinuata, Medicago marina, Pancratium maritimum, and Silene canescens. Seeds were collected in sand dunes along the Tyrrhenian coasts in south Tuscany and north Latium (central Italy). The germination ability was tested at the Tuscia Germplasm Bank (BGT) at six constant temperatures (from 5 to $30^{\circ} \mathrm{C}$ ), under both light (with a $12 / 12 \mathrm{~h}$ photoperiod) and total dark. Our results show a high germination ability and germination rate for the tested species.
\end{abstract}

Key words: coastal dunes, Latium, photoinhibition, psammophytes, Tuscany.

Mediterranean coastal dunes are dynamic and threatened environments, particularly vulnerable being subjected to the strong impact of human activities and in need of conservation actions. Research focused on the assessment of germination requirements of dune species are carried out at the Tuscia Germplasm Bank where over one hundred seed accessions of thirty species collected in Central Italy dunes are long term preserved. Here we present successful germination protocols for six species occurring in the Tyrrhenian sandy dunes: Centaurea sphaerocephala L. subsp. sphaerocephala, Medicago marina L., Pancratium maritimum L., and new data for Italy of Jacobaea maritima (L.) Pelser \& Meijden subsp. maritima, Matthiola sinuata (L.) W.T.Aiton, and Silene canescens Ten.

15. Centaurea sphaerocephala L. subsp. sphaerocephala (Asteraceae) (Fig. 1a)

\section{Accession data}

It: Latium. Tarquinia (Viterbo), S. Agostino (WGS84: $42.173710^{\circ} \mathrm{N}, 11.738198^{\circ} \mathrm{E}$ ), duna costiera, 3 m a.s.1., 4 Sept 2017, S. Magrini \& D. Barreca (BGT-A-43017, Tuscia Germplasm Bank). 


\section{Germination data}

Pre-treatments: sterilization with a solution of 5\% sodium hypochlorite + Tween 20 for 5 minutes, followed by 3 rinses in sterile distilled water.

Germination medium: 1\% agar.

Sample size: 100 achenes for each test $(20 \times 5$ replicates $)$.

\begin{tabular}{|c|c|c|c|c|c|c|}
\hline Germination & Thermoperiod & $\begin{array}{c}\text { Photoperiod } \\
\text { [light/dark] }\end{array}$ & $\mathbf{T}_{\mathbf{1}}$ [d] & $\mathbf{T}_{\mathbf{5 0}}$ [d] & $\mathbf{T}_{\text {max }}$ [d] & MTG [d] \\
\hline $\mathbf{1 0 0} \%$ & constant $25^{\circ} \mathrm{C}$ & $0 / 24 \mathrm{~h}$ & 1.0 & 2.4 & 7.7 & 2.9 \\
\hline $\mathbf{1 0 0} \%$ & constant $25^{\circ} \mathrm{C}$ & $12 / 12 \mathrm{~h}$ & 1.0 & 3.4 & 9.0 & 3.9 \\
\hline $\mathbf{1 0 0} \%$ & constant $20^{\circ} \mathrm{C}$ & $12 / 12 \mathrm{~h}$ & 1.0 & 1.8 & 7.7 & 3.3 \\
\hline $\mathbf{1 0 0} \%$ & constant $20^{\circ} \mathrm{C}$ & $0 / 24 \mathrm{~h}$ & 1.0 & 3.5 & 8.7 & 3.9 \\
\hline $\mathbf{9 6 . 5} \%$ & constant $15^{\circ} \mathrm{C}$ & $0 / 24 \mathrm{~h}$ & 4.0 & 3.7 & 9.7 & 4.9 \\
\hline $\mathbf{9 5 . 0} \%$ & constant $15^{\circ} \mathrm{C}$ & $12 / 12 \mathrm{~h}$ & 4.0 & 3.8 & 8.7 & 4.9 \\
\hline $\mathbf{9 1 . 7 \%}$ & constant $30^{\circ} \mathrm{C}$ & $12 / 12 \mathrm{~h}$ & 1.0 & 2.6 & 11.7 & 4.1 \\
\hline $\mathbf{9 0 . 0} \%$ & constant $30^{\circ} \mathrm{C}$ & $0 / 24 \mathrm{~h}$ & 1.0 & 2.4 & 12.0 & 3.5 \\
\hline $\mathbf{8 8 . 0} \%$ & constant $10^{\circ} \mathrm{C}$ & $0 / 24 \mathrm{~h}$ & 5.0 & 7.1 & 17.0 & 7.6 \\
\hline
\end{tabular}

\section{Observations}

Centaurea sphaerocephala is a perennial species of foredunes and fixed dunes, quite common in Central and South Italy (Acosta \& Ercole 2015). High germination (> 72\%) was obtained at a wide range of temperatures $\left(10-30^{\circ} \mathrm{C}\right)$, independently from the applied light/dark conditions. Lower germination percentages have been reported for this species, $79 \%$ by Menini \& al. (2014) for Tuscany (Italy) and $82 \%$ by Royal Botanic Gardens Kew (2019), both at $20^{\circ} \mathrm{C}$ under an $8 / 16$ h photoperiod.

16. Jacobaea maritima (L.) Pelser \& Meijden subsp. maritima (Asteraceae) (Fig. 1b)

\section{Accession data}

It: Tuscany. Grosseto (Grosseto), loc. Collelungo, Marina di Alberese (WGS84: $\left.42.638073^{\circ} \mathrm{N}, 11.067104^{\circ} \mathrm{E}\right)$, duna costiera, $3 \mathrm{~m}$ a.s.1., 30 Jun 2017, S. Magrini \& D. Barreca (BGT-A-41717, Tuscia Germplasm Bank). 


\section{Germination data}

Pre-treatments: sterilization with a solution of 5\% sodium hypochlorite + Tween 20 for 5 minutes followed by 3 rinses in sterile distilled water.

Germination medium: 1\% agar.

Sample size: 100 achenes for each test $(25 \times 4$ replicates $)$.

\begin{tabular}{|c|c|c|c|c|c|c|}
\hline Germination & Thermoperiod & $\begin{array}{c}\text { Photoperiod } \\
{[\text { light/dark] }}\end{array}$ & $\mathbf{T}_{\mathbf{1}}[\mathbf{d}]$ & $\mathbf{T}_{\mathbf{5 0}}[\mathbf{d}]$ & $\mathbf{T}_{\mathbf{m a x}}[\mathbf{d}]$ & $\mathbf{M T G}[\mathbf{d}]$ \\
\hline $\mathbf{8 1 . 5} \%$ & constant $15^{\circ} \mathrm{C}$ & $12 / 12 \mathrm{~h}$ & 4.3 & 6.6 & 21.0 & 8.4 \\
\hline
\end{tabular}

\section{Observations}

Jacobaea maritima subsp. maritima is a perennial dune species with a West Mediterranean distribution. Here we report the first germination data for this species for Italy. Germination percentages higher than $70 \%$ were obtained only at $15-25^{\circ} \mathrm{C}$ in the light, with the lowest percentages at $30^{\circ} \mathrm{C}$ in the dark $(14.6 \%)$ and at $5^{\circ} \mathrm{C}$ in the light (18.9\%). Light-promoted seed germination highlighted by Van der Meijden \& Van der Waals-Kooi (1979) for a Dutch population of Senecio jacobaea L. can be confirmed in this species only at temperatures higher than $15^{\circ} \mathrm{C}$, while photoinhibition was found at $10^{\circ} \mathrm{C}$ and $5^{\circ} \mathrm{C}$.

17. Matthiola sinuata (L.) W.T.Aiton (Brassicaceae) (Fig. 1c)

\section{Accession data}

It: Latium. Tarquinia (Viterbo), S. Agostino (WGS84: $42.173365^{\circ} \mathrm{N}, 11.738726^{\circ} \mathrm{E}$ ), duna costiera, 2 m a.s.1., 4 Sept 2017, S. Magrini \& D. Barreca (BGT-A-43117, Tuscia Germplasm Bank).

It: Tuscany. Castiglione della Pescaia (Grosseto), loc. Roccamare, presso il fosso Tonfone (WGS84: $42.773051^{\circ} \mathrm{N}, 10.823768^{\circ} \mathrm{E}$ ), duna costiera, $1 \mathrm{~m}$ a.s.1., $31 \mathrm{Aug}$ 2014, S. Magrini (BGT-A-31114, Tuscia Germplasm Bank).

\section{Germination data}

Pre-treatments: sterilization with a solution of 5\% sodium hypochlorite + Tween 20 for 5 minutes followed by 3 rinses in sterile distilled water.

Germination medium: 1\% agar.

Sample size: 100 seeds for each test $(20 \times 5$ replicates $)$. 


\begin{tabular}{|c|c|c|c|c|c|c|c|}
\hline Germination & Thermoperiod & $\begin{array}{c}\text { Photoperiod } \\
{[\text { light/dark] }}\end{array}$ & $\mathbf{T}_{\mathbf{1}}[\mathbf{d}]$ & $\mathbf{T}_{\mathbf{5 0}}[\mathbf{d}]$ & $\mathbf{T}_{\max }[\mathbf{d}]$ & MTG [d] & Accession code \\
\hline $\mathbf{9 0 . 7} \%$ & constant $25^{\circ} \mathrm{C}$ & $12 / 12 \mathrm{~h}$ & 2.0 & 3.6 & 12.0 & 3.8 & BGT-A-43117 \\
\hline $\mathbf{8 6 . 7} \%$ & constant $25^{\circ} \mathrm{C}$ & $12 / 12 \mathrm{~h}$ & 3.0 & 5.3 & 15.0 & 7.6 & BGT-A-31114 \\
\hline $\mathbf{8 5 . 0} \%$ & constant $25^{\circ} \mathrm{C}$ & $0 / 24 \mathrm{~h}$ & 1.7 & 3.6 & 17.3 & 4.3 & BGT-A-31114 \\
\hline
\end{tabular}

\section{Observations}

Matthiola sinuata is a short-lived (biennial) species typical of the sand beach driftlines, occurring in Italy mainly along the Tyrrhenian coasts (Acosta \& Ercole 2015). Here we report the first germination data for this species for Italy. Germination tests using six constant temperature regimes $\left(5-30^{\circ} \mathrm{C}\right)$ showed that $25^{\circ} \mathrm{C}$ is the optimum temperature for both the accessions, the same temperature reported also by Pérez-García $\&$ al. (2007) for Spain. High germination $(>70 \%)$ was obtained only at warmer temperatures $\left(20-30^{\circ} \mathrm{C}\right)$, independently from the applied photoperiod, unlike Royal Botanic Gardens Kew (2019) which reported the highest percentage $(90 \%)$ at $11^{\circ} \mathrm{C}$ and at $25^{\circ} \mathrm{C}$ a lower percentage (78\%) than those reported here.

Strong photoinhibition has been found in other dune species of the family Brassicaceae, like Matthiola tricuspidata (L.) R.Br., Cakile maritima Scop., and Malcolmia littorea (L.) R.Br. (Thanos \& al. 1991, 1994; De Vitis \& al. 2014, 2018; Carta \& al. 2017). On the other hand, our results highlighted a strong photoinhibition of seed germination in $M$. sinuata exclusively at temperatures below $10^{\circ} \mathrm{C}$ and only a weak effect at $15-20^{\circ} \mathrm{C}$.

18. Medicago marina L. (Fabaceae) (Fig. 1d)

\section{Accession data}

It: Tuscany. Grosseto (Grosseto), loc. Rosmarina, Marina di Grosseto (WGS84: $42.726583^{\circ} \mathrm{N}, 10.969202^{\circ} \mathrm{E}$ ), duna costiera, $4 \mathrm{~m}$ a.s.1., 24 Jul 2016, S. Magrini (BGT-A-39416, Tuscia Germplasm Bank).

\section{Germination data}

Pre-treatments: manual scarification by sandpaper; washing with sterile distilled water + Tween 20 for 5 minutes followed by 3 rinses in sterile distilled water.

Germination medium: 1\% agar.

Sample size: 100 seeds for each test $(20 \times 5$ replicates $)$. 


\begin{tabular}{|c|c|c|c|c|c|c|}
\hline Germination & Thermoperiod & $\begin{array}{c}\text { Photoperiod } \\
{[\text { light/dark] }}\end{array}$ & $\mathbf{T}_{\mathbf{1}}[\mathbf{d}]$ & $\mathbf{T}_{\mathbf{5 0}}[\mathbf{d}]$ & $\mathbf{T}_{\mathbf{m a x}}[\mathbf{d}]$ & $\mathbf{M T G}[\mathbf{d}]$ \\
\hline $\mathbf{1 0 0} \%$ & constant $20^{\circ} \mathrm{C}$ & $12 / 12 \mathrm{~h}$ & 5.0 & 5.9 & 20.3 & 9.6 \\
\hline $\mathbf{1 0 0} \%$ & constant $30^{\circ} \mathrm{C}$ & $0 / 24 \mathrm{~h}$ & 1.7 & 8.1 & 43.7 & 20.5 \\
\hline $\mathbf{1 0 0} \%$ & constant $5^{\circ} \mathrm{C}$ & $0 / 24 \mathrm{~h}$ & 10.0 & 16.9 & 44.7 & 21.1 \\
\hline $\mathbf{9 7 . 6 \%}$ & constant $5^{\circ} \mathrm{C}$ & $12 / 12 \mathrm{~h}$ & 13.3 & 16.3 & 67.3 & 24.6 \\
\hline $\mathbf{9 5 . 2} \%$ & constant $30^{\circ} \mathrm{C}$ & $12 / 12 \mathrm{~h}$ & 3.3 & 6.8 & 28.0 & 10.7 \\
\hline $\mathbf{9 2 . 6 \%}$ & constant $10^{\circ} \mathrm{C}$ & $12 / 12 \mathrm{~h}$ & 8.3 & 11.1 & 41.0 & 18.8 \\
\hline $\mathbf{9 0 . 5} \%$ & constant $20^{\circ} \mathrm{C}$ & $0 / 24 \mathrm{~h}$ & 2.0 & 1.9 & 39.3 & 6.9 \\
\hline $\mathbf{8 8 . 1} \%$ & constant $10^{\circ} \mathrm{C}$ & $0 / 24 \mathrm{~h}$ & 5.0 & 5.6 & 18.0 & 13.3 \\
\hline $\mathbf{8 8 . 1 \%}$ & constant $15^{\circ} \mathrm{C}$ & $0 / 24 \mathrm{~h}$ & 2.3 & 2.8 & 39.0 & 7.2 \\
\hline $\mathbf{8 7 . 5 \%}$ & constant $15^{\circ} \mathrm{C}$ & $12 / 12 \mathrm{~h}$ & 5.0 & 5.5 & 25.0 & 9.0 \\
\hline $\mathbf{8 6 . 1 \%}$ & constant $25^{\circ} \mathrm{C}$ & $0 / 24 \mathrm{~h}$ & 1.0 & 2.0 & 7.0 & 3.3 \\
\hline $\mathbf{8 3 . 9 \%}$ & constant $25^{\circ} \mathrm{C}$ & $12 / 12 \mathrm{~h}$ & 3.0 & 4.9 & 36.3 & 11.4 \\
\hline
\end{tabular}

\section{Observations}

Medicago marina is a perennial species characteristic of the embryonic shifting dunes, common along the Italian coasts (Acosta \& Ercole 2015). As for many other genera of Fabaceae, Medicago seeds are characterized by a physical dormancy due to the impermeable coat. In accordance with Scippa \& al. (2011), mechanical scarification by sandpaper improved seed germination, with high percentages $(\geq 83.9 \%)$ at all the tested conditions (six constant temperatures from 5 to $30^{\circ} \mathrm{C}$, both in the light and in the dark). In particular, the fastest germination was recorded at $25^{\circ} \mathrm{C}$ in darkness $\left(\mathrm{T}_{1}=1\right.$ day, $\mathrm{T}_{50}=2$ days and a total of 7 days for the maximum germination), even if with a lower germination percentage than reported, under the same conditions, by Scippa \& al. (2011) for seeds from Adriatic coastal dunes (south Italy). The slowest germination was recorded at $5^{\circ} \mathrm{C}$ under light condition.

19. Pancratium maritimum L. (Amaryllidaceae) (Fig. 1e)

\section{Accession data}

It: Latium. Tarquinia (Viterbo), loc. Saline (WGS84: $42.210943^{\circ} \mathrm{N}, 11.708386^{\circ} \mathrm{E}$ ), 
duna costiera, 2 m a.s.1., 25 Sept 2016, A. Caldelli \& S. Magrini (BGT-A-39816, Tuscia Germplasm Bank).

\section{Germination data}

Pre-treatments: sterilization with a solution of 5\% sodium hypochlorite + Tween 20 for 5 minutes followed by 3 rinses in sterile distilled water.

Germination medium: 1\% agar.

Sample size: 60 seeds for each test $(20 \times 3$ replicates $)$.

\begin{tabular}{|c|c|c|c|c|c|c|}
\hline Germination & Thermoperiod & $\begin{array}{c}\text { Photoperiod } \\
{[\text { light/dark] }}\end{array}$ & $\mathbf{T}_{\mathbf{1}}[\mathbf{d}]$ & $\mathbf{T}_{\mathbf{5 0}}[\mathbf{d}]$ & $\mathbf{T}_{\mathbf{m a x}}[\mathbf{d}]$ & MTG [d] \\
\hline $\mathbf{9 7 . 8 \%}$ & constant $20^{\circ} \mathrm{C}$ & $0 / 24 \mathrm{~h}$ & 12.0 & 15.3 & 23.7 & 14.9 \\
\hline $\mathbf{9 7 . 8 \%}$ & constant $15^{\circ} \mathrm{C}$ & $12 / 12 \mathrm{~h}$ & 20.3 & 36.0 & 55.3 & 35.7 \\
\hline $\mathbf{8 8 . 9 \%}$ & constant $15^{\circ} \mathrm{C}$ & $0 / 24 \mathrm{~h}$ & 24.3 & 32.3 & 58.3 & 31.9 \\
\hline $\mathbf{8 2 . 2} \%$ & constant $30^{\circ} \mathrm{C}$ & $0 / 24 \mathrm{~h}$ & 14.3 & 23.9 & 63.3 & 27.7 \\
\hline
\end{tabular}

\section{Observations}

Pancratium maritimum is a species of coastal dunes, with a wide distribution along the Italian sandy coasts even if overcollection, urbanization, and tourism put serious threats to the species, resulting in a significant decrease of its populations (De Castro $\&$ al. 2012; Giovino \& al. 2015). To date, these are the best germination data for this species for Latium. Our germination tests defined an optimal temperature range of 15$20^{\circ} \mathrm{C}$, in accordance with data previously reported for Sardinia $\left(95-98 \%\right.$ at $15-20^{\circ} \mathrm{C}$, in the dark; Bacchetta \& al. 2007). Moreover, at all the temperatures in the dark (except $20^{\circ} \mathrm{C}$ ), higher germination percentages have been recorded than those reported by Balestri \& Cinelli (2004) for Tuscany (53\% vs. $0 \%$ at $5^{\circ} \mathrm{C}, 80 \%$ vs. $25 \%$ at $10^{\circ} \mathrm{C}, 89 \%$ vs. $50 \%$ at $15^{\circ} \mathrm{C}$, and $82 \%$ vs. $25 \%$ at $30^{\circ} \mathrm{C}$ ) and by De Lillis $\&$ al. (2004) for Latium $\left(60 \%\right.$ with a longer $\mathrm{T}_{1}$ at $22 / 15^{\circ} \mathrm{C}$ with a $14 / 10 \mathrm{~h}$ photoperiod).

Our results, however, highlighted the ability of $P$. maritimum seeds to germinate in a wide range of temperatures $\left(5-30^{\circ} \mathrm{C}\right)$ both in the dark (53-98\%) and in the light (7$98 \%$ ). Moreover, we observed a strong photoinhibition of seed germination exclusively at $5^{\circ} \mathrm{C}$, with seeds weakly photoinhibited at $\mathrm{T}>20^{\circ} \mathrm{C}$, in accordance with Carta \& al. (2017), and with no inhibition at $10-15^{\circ} \mathrm{C}$. 
20. Silene canescens Ten. (Caryophyllaceae) (Fig. 1f)

\section{Accession data}

It: Latium. Montalto di Castro (Viterbo), Montalto Marina (WGS84: $42.321758^{\circ} \mathrm{N}$, $11.585935^{\circ} \mathrm{E}$ ), duna costiera, $3 \mathrm{~m}$ a.s.1., 12 May 2016, S. Magrini \& D. Barreca (BGT-A-36516, Tuscia Germplasm Bank).

\section{Germination data}

Pre-treatments: sterilization with a solution of 5\% sodium hypochlorite + Tween 20 for 5 minutes followed by 3 rinses in sterile distilled water.

Germination medium: 1\% agar.

Sample size: 100 seeds for each test $(20 \times 5$ replicates $)$.

\begin{tabular}{|c|c|c|c|c|c|c|}
\hline Germination & Thermoperiod & $\begin{array}{c}\text { Photoperiod } \\
{[\text { light/dark] }}\end{array}$ & $\mathbf{T}_{\mathbf{1}}[\mathbf{d}]$ & $\mathbf{T}_{\mathbf{5 0}}[\mathbf{d}]$ & $\mathbf{T}_{\max }[\mathbf{d}]$ & $\mathbf{M T G}[\mathbf{d}]$ \\
\hline $\mathbf{1 0 0} \%$ & constant $20^{\circ} \mathrm{C}$ & $12 / 12 \mathrm{~h}$ & 1.3 & 1.3 & 6.0 & 2.4 \\
\hline $\mathbf{9 8 . 3 \%}$ & constant $20^{\circ} \mathrm{C}$ & $0 / 24 \mathrm{~h}$ & 1.0 & 1.3 & 11.3 & 2.3 \\
\hline $\mathbf{9 8 . 3 \%}$ & constant $25^{\circ} \mathrm{C}$ & $0 / 24 \mathrm{~h}$ & 1.0 & 0.5 & 5.3 & 1.4 \\
\hline $\mathbf{9 6 . 7 \%}$ & constant $25^{\circ} \mathrm{C}$ & $12 / 12 \mathrm{~h}$ & 1.0 & 0.7 & 3.3 & 1.4 \\
\hline $\mathbf{9 5 . 0} \%$ & constant $15^{\circ} \mathrm{C}$ & $12 / 12 \mathrm{~h}$ & 1.0 & 1.4 & 16.3 & 2.7 \\
\hline $\mathbf{9 3 . 3 \%}$ & constant $15^{\circ} \mathrm{C}$ & $0 / 24 \mathrm{~h}$ & 1.0 & 0.7 & 9.3 & 1.7 \\
\hline $\mathbf{9 1 . 7 \%}$ & constant $10^{\circ} \mathrm{C}$ & $0 / 24 \mathrm{~h}$ & 6.0 & 5.6 & 15.0 & 6.7 \\
\hline $\mathbf{9 0 . 2} \%$ & constant $30^{\circ} \mathrm{C}$ & $0 / 24 \mathrm{~h}$ & 1.0 & 0.5 & 3.0 & 1.1 \\
\hline $\mathbf{9 0 . 0} \%$ & constant $30^{\circ} \mathrm{C}$ & $12 / 12 \mathrm{~h}$ & 1.0 & 0.6 & 10.3 & 1.9 \\
\hline
\end{tabular}

\section{Observations}

Silene canescens (= S. colorata Poir.) is an annual Mediterranean species of foredunes and fixed dunes, quite common along the Italian sandy coasts (Acosta \& Ercole 2015). Here we report the first germination data for this species for Italy. Successful and fast ger- 
mination $\left(>90 \%, \mathrm{~T}_{50}=0.5-1.4\right)$ was obtained at a wide range of constant temperatures (15$30^{\circ} \mathrm{C}$ ), in accordance with Royal Botanic Gardens Kew (2019), with germination percentages much higher than those reported for Portugal: $0 \%$ at $15-20^{\circ} \mathrm{C}$ and $28 \%$ at $25^{\circ} \mathrm{C}$, under light condition (Marques \& al. 2007). The lowest germination percentages were recorded at $5^{\circ} \mathrm{C}$, where strong photoinhibition of seed germination was observed (Carta \& al. 2017).

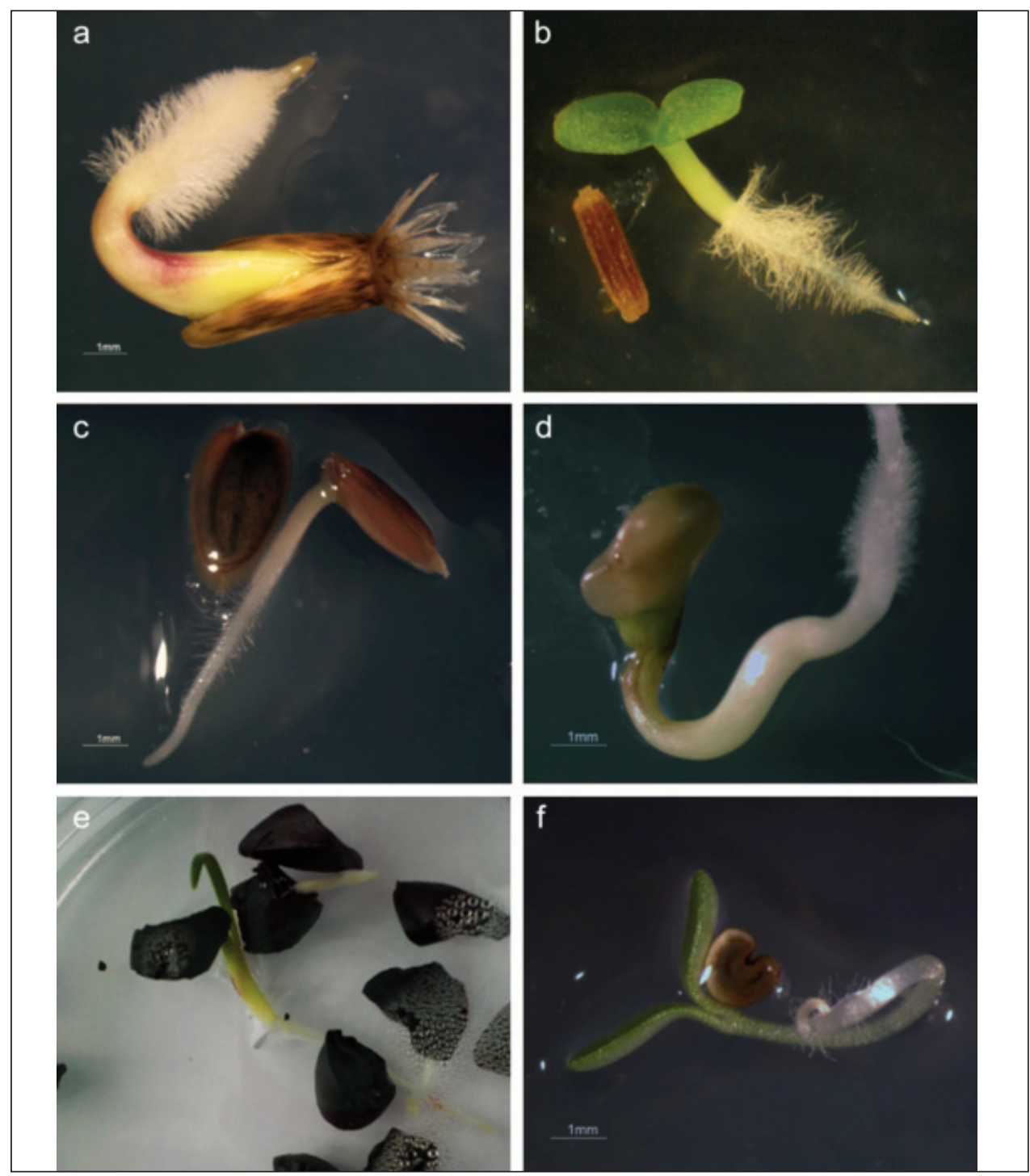

Fig. 1. Germinated seeds of: a, Centaurea sphaerocephala subsp. sphaerocephala; b, Jacobaea maritima subsp. maritima; c, Matthiola sinuata; d, Medicago marina; e, Pancratium maritimum; f, Silene canescens. 


\section{References}

Acosta, A. T. R. \& Ercole, S. (eds) 2015: Gli habitat delle coste sabbiose italiane: ecologia e problematiche di conservazione. - ISPRA, Serie Rapporti, 215/2015.

Bacchetta, G., Fenu, G., Mattana, E., Meloni, F. \& Podda, L. 2007: Conservazione ex situ e in situ della biodiversità vegetale dell'Area Marina Protetta di Capo Carbonara (Sardegna sud-orientale). - Fitosociologia 44(1): 157-164.

Balestri, E. \& Cinelli, F. 2004: Germination and early-seedling establishment capacity of Pancratium maritimum L. (Amaryllidaceae) on coastal dunes in the North-Western Mediterranean. - J. Coast. Res. 20(3): 761-770. https://doi.org/10.2112/1551-5036(2004)20[761:gaeeco]2.0.co;2

Carta, A., Skourti, E., Mattana, E., Vandelook, F. \& Thanos, C. 2017: Photoinhibition of seed germination: Occurrence, ecology and phylogeny. - Seed Sci. Res. 27(2): 131-153. https://doi.org/10.1017/s0960258517000137

De Castro, O., Brullo, S., Colombo, P., Jury, S., De Luca, P. \& Di Maio, A. 2012: Phylogenetic and biogeographical inferences for Pancratium (Amaryllidaceae), with an emphasis on the Mediterranean species based on plastid sequence data. - Bot. J. Linn. Soc. 170: 12-28. https://doi.org/10.1111/j.1095-8339.2012.01268.x

De Lillis, M., Costanzo, L., Bianco, P. M. \& Tinelli, A. 2004: Sustainability of sand dune restoration along the coast of the Tyrrhenian Sea. - J. Coast. Conserv. 10(1): 93-100. https://doi.org/10.1652/1400-0350(2004)010[0093:sosdra]2.0.co;2

De Vitis, M., Mattioni, C., Mattana, E., Pritchard, H. W., Seal, C., Ulian, T., Cherubini, M. \& Magrini, S., 2018: Integration of genetic and seed fitness data to the conservation of isolated subpopulations of the Mediterranean plant Malcolmia littorea - P1. Biol. 20(S1): 203-213. https://doi.org/10.1111/plb.12637

—, Seal, C., Ulian, T., Pritchard, H. W., Magrini, S., Fabrini, G. \& Mattana, E. 2014: Rapid adaptation of seed germination requirements of the threatened Mediterranean species Malcolmia littorea (Brassicaceae) and implications for its reintroduction - S. Afr. J. Bot. 94: 46-50. https://doi.org/10.1016/j.sajb.2014.05.008

Giovino, A., Domina, G., Bazan, G., Campisi, P. \& Scibetta, S. 2015: Taxonomy and conservation of Pancratium maritimum (Amaryllidaceae) and relatives in the Central Mediterranean. - Acta Bot. Gallica 162(4): 289-299. https://doi.org/10.1080/12538078.2015.1089416

Marques, I., Draper, D. \& Martins-Loução, M. A. 2007: Influence of temperature on seed germination in seven Mediterranean grassland species from SE Portugal. - Bocconea 21: 367-372.

Meijden van der, E. \& Waals-Kooi van der, R. E. 1979: The population ecology of Senecio jacobaea in a sand dune system. I. Reproductive strategy and the biennial habit. - J. Ecol. 67: 131153. https://doi.org/10.2307/2259341

Menini, S., Borzatti de Loewenstern, A. \& Silvi, E. 2014: Test di germinazione su specie rare o localmente estinte conservate nella banca del germoplasma del Museo di Storia Naturale del Mediterraneo: taxa indagati Centaurea spp. e Halimium halimifolium (L.) Willk. - Museol. Sci. Mem. 11/2014: 201-204. https://doi.org/10.5962/bhl.part.10419

Pérez-García, F., González-Benito, M. E. \& Gómez-Campo, C. 2007: High viability recorded in ultra-dry seeds of 37 species of Brassicaceae after almost 40 years of storage. - Seed Sci. \& Technol. 35: 143-153. https://doi.org/10.15258/sst.2007.35.1.13

Royal Botanic Gardens Kew. 2019: Seed Information Database (SID). Version 7.1. Available from: http://data.kew.org/sid/ [Last Accessed 10.10.2019].

Scippa, G. S., Petrollini, E., Trupiano, D., Rocco, M., Falco, G., Di Michele, M. \& Chiatante, D. 2011: Dormancy of Medicago marina (L.) seed. - Environ. Exp. Bot. 72: 320-329. https://doi.org/10.1016/j.envexpbot.2011.04.005 
Thanos, C. A., Georghiou, K. \& Delipetrou, P. 1994: Photoinhibition of seed germination in the maritime plant Matthiola tricuspidata. - Ann. Bot. 73: 639-644. https://doi.org/10.1006/ anbo.1994.1080

—, - , Douma, D. J. \& Marangaki, C. J. 1991: Photoinhibition of seed germination in Mediterranean maritime plants. - Ann. Bot. 68: 469-475. https://doi.org/10.1093/oxfordjournals.aob.a088280

Addresses of the authors:

Sara Magrini ${ }^{1}$, Donatella Barreca ${ }^{1}$ Ludovica Neri ${ }^{1}$, Andrea Iacomino ${ }^{1}$, Francesco Barbarese $^{1}$, Sara Superchi ${ }^{1} \&$ Laura Zucconi ${ }^{1,2}$,

${ }^{1}$ Tuscia Germplasm Bank, Tuscia University, Viterbo, Italy. E-mails: magrini@unitus.it; barreca@unitus.it

${ }^{2}$ Department of Ecological and Biological Sciences, Tuscia University, Viterbo, Italy. E-mail: zucconi@unitus.it 